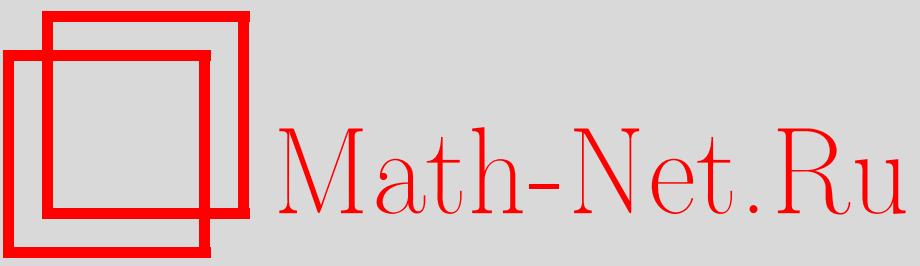

А. А. Голдаева, Об аналоге характеризационной теоремы Леви для случайного блуждания, УМH, 2004, том 59, выпуск 2, 191-192

DOI: https://doi.org/10.4213/rm727

Использование Общероссийского математического портала Math-Net.Ru подразумевает, что вы прочитали и согласны с пользовательским соглашением

http://www.mathnet.ru/rus/agreement

Параметры загрузки:

IP: 54.237 .59 .107

26 апреля 2023 г., 09:14:33 


\title{
ОБ АНАЛОГЕ ХАРАКТЕРИЗАЦИОННОЙ ТЕОРЕМЫ ЛЕВИ ДЛЯ СЛУЧАЙНОГО БЛУЖДАНИЯ
}

\author{
А. А. ГОЛДАЕВА
}

Пусть $(B(t))_{t \geqslant 0}-$ броуновское движение на некотором вероятностном пространстве $(\Omega, \mathscr{F}, \mathrm{P})$, $M(t)=\sup _{s \leqslant t} B(s), L_{t}(B)$ - локальное время броуновского движения. Теорема Леви утверждает (см., например, $[1 ;$ гл. $6, \S 2])$, что $(M-B, M) \stackrel{\text { law }}{=}(|B|, L(B))$. Известно обобщение этой теоремы, полученное С.Э. Граверсеном и А.Н. Ширяевьм для броуновского движения со сносом, а именно, если $B^{\lambda}=B(t)+\lambda t, M^{\lambda}(t)=\sup _{s \leqslant t} B^{\lambda}(s)$, то $\left(M^{\lambda}-B^{\lambda}, M^{\lambda}\right) \stackrel{\text { law }}{=}\left(\left|X^{\lambda}\right|, L\left(X^{\lambda}\right)\right)$, где $\left(X_{t}^{\lambda}\right)_{0 \leqslant t \leqslant 1}$ - единственное строгое решение стохастического дифференциального уравнения $d X_{t}^{\lambda}=-\lambda \operatorname{sign} X_{t}^{\lambda} d t+d B_{t}, X_{0}^{\lambda}=0$, a $L_{t}\left(X^{\lambda}\right)=\lim _{\varepsilon \downarrow 0} \frac{1}{2 \varepsilon} \int_{0}^{t} \mathrm{I}\left\{\left|X_{s}^{\lambda}\right| \leqslant \varepsilon\right\} d s$.

В данной работе показывается, что (вопреки мнению, что сформулированные результаты присущи лишь броуновскому движению) аналогичные результаты справедливы и для случайных блужданий, порожденных схемой Бернулли.

Пусть $\xi_{1}, \xi_{2}, \ldots$ - случайные величины на вероятностном пространстве $(\Omega, \mathscr{F}, \mathrm{P})$, принимающие значения \pm 1 с вероятностью $\frac{1}{2}$, и $S_{n}=\xi_{1}+\cdots+\xi_{n}$. Обозначим $M_{n}=\max _{k \leqslant n} S_{k}$.

Теорема 1. Имеет место следующее свойство по распределению: $\operatorname{Law}\left(M_{n}-S_{n}, M_{n}\right.$; $n \geqslant 0)=\operatorname{Law}\left(\left|S_{n}\right|, L_{n}(S) ; n \geqslant 0\right)$, где $L_{n}(S)=\#\left\{k: 1 \leqslant k \leqslant n: S_{k-1}=0\right\}-$ число нулей последовательности $S_{n}$.

ДоказАтельство. Рассмотрим дискретный аналог формулы Танака (см. [3; гл. 2, §1b])

$$
\left|S_{n}\right|=\sum_{k=1}^{n}\left(\operatorname{sign} S_{k-1}\right) \Delta S_{k}+L_{n}(S)
$$

Обозначим $A_{n}=\sum_{k=1}^{n}\left(\operatorname{sign} S_{k-1}\right) \Delta S_{k}$. Перепишем формулу (1) в виде $\left|S_{n}\right|=A_{n}+L_{n}(S)$ и применим к ней лемму Скорохода (см. $[1 ;$ гл. $6, \S 2])$. Тогда $L_{n}(S)=\sup _{k \leqslant n} S_{k}$. С другой стороны, $M_{n}-S_{n}=-S_{n}+M_{n}$. Таким образом, видим, что $M$ и $M-S$ (соответственно $L$ и $|S|$ ) могут быть получены из $-S$ (соответственно из $S$ ) с помощью леммы Скорохода одной и той же детерминированной процедурой. Так как $\operatorname{Law}(-S)=\operatorname{Law}(A)$, то теорема доказана.

Рассмотрим теперь несимметричный случай. Пусть $\eta_{1}, \eta_{2}, \ldots$ - случайные величины на $(\Omega, \mathscr{F}, \mathrm{P})$, принимающие значения \pm 1 с вероятностями $p$ и $q=1-p$ соответственно, $\widetilde{S}_{n}=\eta_{1}+$ $\cdots+\eta_{n}, \widetilde{M}_{n}=\max _{k \leqslant n} \widetilde{S}_{k}$.

Теорема 2. Имеет место следующее равенство по распределению:

$$
\operatorname{Law}\left(\widetilde{M}_{n}-\widetilde{S}_{n}, \widetilde{M}_{n} ; n \geqslant 0\right)=\operatorname{Law}\left(\left|X_{n}\right|, L_{n}(X) ; n \geqslant 0\right),
$$

где $\Delta X_{n}=-\operatorname{sign} X_{n-1}+\Delta S_{n}, X_{0}=0, L_{n}(X)=\#\left\{k: 1 \leqslant k \leqslant n: X_{k-1}=0\right\}$.

ДокАЗАТЕЛЬСтво. Определим на $(\Omega, \mathscr{F}, \mathrm{P})$ новую меру Р

$$
\frac{d \widetilde{\mathrm{P}}}{d \mathrm{P}}(s)=\prod_{k=1}^{n}(2 p)^{\frac{1}{2}\left(1+s_{k}-s_{k-1}\right)}(2 q)^{\frac{1}{2}\left(1-\left(s_{k}-s_{k-1}\right)\right)} .
$$

По теореме Гирсанова $\operatorname{Law}(\widetilde{S} \mid \widetilde{\mathrm{P}})=\operatorname{Law}(S \mid \mathrm{P})$. Пусть $G(x, y)$ - непрерьвный функционал из $D(\mathbb{R}) \times D(\mathbb{R})$ в $\mathbb{R}$. Используя теорему 1 , получаем

$$
\mathrm{E}[G(\widetilde{M}-\widetilde{S}, \widetilde{M})]=\widetilde{\mathrm{E}}\left[\left(\frac{d \widetilde{\mathrm{P}}}{d \mathrm{P}}(\widetilde{S})\right)^{-1} G(\widetilde{M}-\widetilde{S}, \widetilde{M})\right]=\mathrm{E}\left[\left(\frac{d \widetilde{\mathrm{P}}}{d \mathrm{P}}(L-|S|)\right)^{-1} G(|S|, L(S))\right]
$$


С другой стороны, введем новую меру $\mathrm{P}^{*}$ :

$$
\begin{aligned}
\frac{d \mathrm{P}^{*}}{d \mathrm{P}}(x)=\prod_{k=1}^{n}(2 p)^{\frac{1}{2}\left(1+\left(-\sum_{j=1}^{k}\left(\operatorname{sign} x_{j-1}\right) \Delta x_{j}+\sum_{j=1}^{k-1}\left(\operatorname{sign} x_{j-1}\right) \Delta x_{j}\right)\right)} & \\
& \times(2 q)^{\frac{1}{2}\left(1-\left(-\sum_{j=1}^{k}\left(\operatorname{sign} x_{j-1}\right) \Delta x_{j}+\sum_{j=1}^{k-1}\left(\operatorname{sign} x_{j-1}\right) \Delta x_{j}\right)\right)} .
\end{aligned}
$$

По теореме Гирсанова $\operatorname{Law}\left(X \mid \mathrm{P}^{*}\right)=\operatorname{Law}(S \mid \mathrm{P})$. Используя формулу (1), получаем

$$
\begin{aligned}
\mathrm{E}[G(|X|, L(X))] & =\mathrm{E}^{*}\left[\left(\frac{d \mathrm{P}^{*}}{d \mathrm{P}}(X)\right)^{-1} G(|X|, L(X))\right] \\
& =\mathrm{E}\left[\left(\frac{d \mathrm{P}^{*}}{d \mathrm{P}}(S)\right)^{-1} G(|S|, L(S))\right]=\mathrm{E}\left[\left(\frac{d \mathrm{P}^{*}}{d \mathrm{P}}(L-|S|)\right)^{-1} G(|S|, L(S))\right] .
\end{aligned}
$$

Сравнивая (3) и (4), получаем (2).

ТеОРема 3. При $x, y \geqslant 0$ выполнено соотношение

$$
\mathrm{P}\left(S_{n}<y-x, M_{n} \geqslant y\right)=\mathrm{P}\left(S_{n}>y+x\right) .
$$

ДокАЗАтЕльство. При $y=0$ (5) превращается в равенство $\mathrm{P}\left(S_{n}<-x\right)=\mathrm{P}\left(S_{n}>x\right)$. Пусть $y>0$. Рассмотрим момент остановки $\tau_{y}=\inf \left\{k \geqslant 0: S_{k} \geqslant y\right\}$ и отраженньй процесс

$$
\widehat{S}_{k}= \begin{cases}S_{k}, & 0 \leqslant k \leqslant \tau_{y}, \\ 2 S_{\tau_{y}}-S_{k}, & k>\tau_{y} .\end{cases}
$$

Введем момент остановки $\sigma_{y}=\inf \left\{k \geqslant 0: \widehat{S_{k}}=y\right\}$ и заметим, что $\sigma_{y}(\omega)=\tau_{y}(\omega)$ для всех $y \geqslant 0, \omega \in\left\{\tau_{y}<\infty\right\}$. Имеем $\left\{\tau_{y} \leqslant n\right\}=\left\{M_{n} \geqslant y\right\}$ для любых $n, y \geqslant 0$. Поэтому для всех $B \in \mathscr{B}(C[0 ;+\infty)), n \geqslant 0$ имеем $\mathrm{P}\left(\tau_{y} \leqslant n, S \in B\right)=\mathrm{P}\left(M_{n} \geqslant y, S \in B\right)=\mathrm{P}(S \in \widehat{B} \cap B)$, где $\widehat{B}=G_{t}^{-1}([y ;+\infty)) \in \mathscr{B}(C[0 ;+\infty)), G_{t} f=\max _{s \leqslant t} f(s)$. Применив принцип отражения, видим, что $\mathrm{P}\left(\sigma_{y} \leqslant n, \widehat{S} \in B\right)=\mathrm{P}(\widehat{S} \in \widehat{B} \cup B)=\mathrm{P}(S \in \widehat{B} \cup B)$, т.e. $\operatorname{Law}\left(\tau_{y}, S\right)=\operatorname{Law}\left(\sigma_{y}, \widehat{S}\right)$. При $y \geqslant 0$ и $k \geqslant \sigma_{y}$ получаем $\widehat{S}_{k}=2 S_{\tau_{y}}-S_{k} \geqslant 2 y-S_{k}$. Тогда при всех $y \geqslant 0$ и $x \in \mathbb{R}$

$$
\begin{aligned}
\mathrm{P}\left(M_{n} \geqslant y, S_{n}<y-x\right) & =\mathrm{P}\left(\sigma_{y} \leqslant n, \widehat{S}_{n}<y-x\right)=\mathrm{P}\left(\sigma_{y} \leqslant n, S_{n}<y-x\right) \\
& =\mathrm{P}\left(\tau_{y} \leqslant n, S_{n}>y+x\right)=\mathrm{P}\left(M_{n} \geqslant y, S_{n}>y+x\right) .
\end{aligned}
$$

Если $x \geqslant 0$, то $\mathrm{P}\left(M_{n} \geqslant y, S_{n}>y+x\right)=\mathrm{P}\left(S_{n}>y+x\right)$ и (6) влечет (5).

СлЕДСТВИЕ. $\mathrm{P}\left(M_{n} \geqslant y\right)=\mathrm{P}\left(\left|S_{n}\right| \geqslant y\right)$ nри всех $y \geqslant 0, n \in \mathbb{N}$.

ДокаЗАТЕЛЬСтво. Возьмем $x=0$ в формуле (5): $\mathrm{P}\left(S_{n}<y, M_{n} \geqslant y\right)=\mathrm{P}\left(S_{n}>y\right)$;

$$
\begin{aligned}
\mathrm{P}\left(M_{n} \geqslant y\right) & =\mathrm{P}\left(M_{n} \geqslant y, S_{n}<y\right)+\mathrm{P}\left(M_{n} \geqslant y, S_{n} \geqslant y\right) \\
& =\mathrm{P}\left(S_{n}>y\right)+\mathrm{P}\left(S_{n} \geqslant y\right)=\mathrm{P}\left(\left|S_{n}\right| \geqslant y\right) .
\end{aligned}
$$

\section{СПИСОК ЛИТЕРАТУРЫ}

[1] D. Revuz, M. Yor. Continuous Martingales and Brownian Motion. Berlin: SpringerVerlag, 1994. [2] S. E. Graversen, A. N. Shiryaev // Bernoulli. 2000. V. 6. № 4. P. 615-620. [3] А. Н. Ширяев. Основы стохастической финансовой математики. Т. 1, 2. М.: Фазис, 1998. 